\title{
Les Comités \\ de protection \\ des personnes
}

Le maillon éthique

de l'encadrement

de la recherche clinique

française

Virginie Rage-Andrieu ${ }^{1}$, François Hirsch ${ }^{2}$

\section{L'encadrement de la recherche clinique en France}

En France, chaque année, des milliers de patients et de volontaires sains acceptent de participer à des recherches biomédicales définies comme l'ensemble des « recherches organisées et pratiquées sur l'être humain en vue du développement des connaissances biologiques ou médicales $»^{1}$. Certaines recherches permettent de tester des produits de santé (médicaments, vaccins, dispositifs médicaux), qui aideront à mieux comprendre les mécanismes responsables des maladies ainsi qu'à évaluer de nouvelles approches thérapeutiques. D'autres recherches ont pour objet de tester de nouvelles pratiques de soin et d'autres, enfin, font appel à des cohortes de patients pour, par exemple, faciliter la recherche de nouveaux biomarqueurs prédictifs de maladies ${ }^{2}$.

Grâce à l'encadrement mis en place par la loi du 20 décembre 1988 relative à la protection des personnes qui se prêtent à des recherches biomédicales (loi Huriet-Sérusclat), modifiée par la loi du 5 mars 2012 relative aux recherches impliquant la personne humaine (loi Jardé) ${ }^{3}$, les participants à ces recherches bénéficient d'une très grande protection. Avec l'entrée en vigueur de la loi Jardé, les études appelées «Recherches impliquant la personne humaine » (RIPH) sont divisées en trois catégories: interventionnelles (RIPH de catégorie 1), interventionnelles à risques et contraintes minimes (RIPH de catégorie 2), non interventionnelles (RIPH de catégorie 3). Les promoteurs ${ }^{4}$

Vignette (Photo (c) Inserm-Patrick Delapierre).

1 Articles L.1121-1 et suivants du code de la santé publique.

2 https://www.coblance.fr/

${ }^{3}$ Loi $n^{\circ} 2012-300$ du 5 mars 2012 relative aux recherches impliquant la personne humaine, JORF, 6 mars 2012, texte 1, https://www.legifrance.gouv.fr/loda/id/JORFTEXT000025441587/2021-03-06/

${ }^{4}$ Le promoteur est une personne physique, une société ou une institution publique ou privée qui prend l'initiative d'un essai clinique et en assume les responsabilités ainsi que le financement.

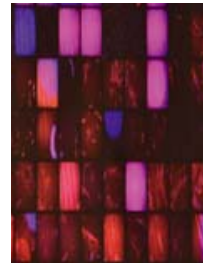

de recherches de catégories 1 doivent demander une autorisation à l'autorité compétente, I'ANSM (Agence nationale de sécurité du médicament et des produits de santé), ou, pour toute recherche
${ }^{1}$ Maître de conférences
en droit de la santé,
Université de Montpellier, UMR 5815, CPP Sud Méditerranée IV, présidente de la Conférence nationale des CPP (CNCP), 34000 Montpellier, France.
${ }^{2}$ Directeur de recherche honoraire Inserm, CPP Île-de- France 7 , membre du bureau de la Conférence nationale des CPP (CNCP),
20 rue Victor-Carmignac,
94110 Arcueil, France.
virginie.rage-andrieu@
umontpellier.fr
francois.hirsch@inserm.fr de catégorie 2 ou 3, doivent uniquement l'informer. En revanche, quelle que soit la catégorie de recherche envisagée, les promoteurs doivent soumettre leur projet à l'évaluation de l'un des comités de protection des personnes (CPP) agréés par le ministre chargé de la santé ${ }^{5}$ et obtenir un avis favorable de ce comité pour que la recherche puisse débuter ${ }^{6}$.

Que sont les CPP ? Comment fonctionnent-ils ? Pourquoi en être membre? Tels sont les points que nous souhaitons aborder.

\section{Les Comités de protection des personnes}

Les Comités de protection des personnes (CPP) sont les héritiers des Comités consultatifs de protection des personnes dans la recherche biomédicale (CCPRB)

\footnotetext{
${ }^{5}$ Article L1123-1 du Code de la santé publique modifié par ordonnance $n^{\circ} 2016$ 800 du 16 juin 2016 - art. 3.

${ }^{6}$ Article L1121-4 CSP https://solidarites-sante.gouv.fr/systeme-de-sante-et-medicosocial/recherche-et-innovation/recherches-impliquant-la-personne-humaine/
} 
instaurés par la loi «Huriet-Sérusclat ». La loi du 9 août $2004^{7}$ fait désormais des CPP des instances qui ne sont plus consultatives, car un avis négatif rendu par les CPP sur une recherche clinique (après cependant une possibilité d'appel de la part du promoteur de l'étude impliquée), empêche celle-ci d'avoir lieu sur le territoire français.

Les CPP sont au nombre de 39, répartis sur l'ensemble du territoire métropolitain; leur composition est pluridisciplinaire; ils comprennent 28 membres experts dans des domaines prédéfinis et répartis au sein de deux collèges ${ }^{8}$, tous nommés par les Agences régionales de santé (ARS). Les membres exercent leurs missions bénévolement, mais reçoivent une indemnité en fonction de leur charge de travail ; ils sont soumis à l'obligation de déclaration publique d'intérêts. Vingt-huit des 39 CPP se sont regroupés au sein d'une association, la Conférence nationale des CPP (CNCP), dont le but est de coordonner, harmoniser, informer, former, améliorer les pratiques, et représenter les CPP auprès des institutions et de tous les acteurs de la recherche?.

Depuis l'accident de janvier 2016, au cours duquel un des six volontaires sains est décédé au cours d'une étude clinique promue par la société Bial $^{10}$, la répartition parmi les 39 CPP des projets de recherche à évaluer se fait par un tirage au sort ${ }^{11}$ organisé par la Commission nationale des recherches impliquant la personne humaine (CNRIPH) ${ }^{12}$. En effet, l'enquête de l'Inspection générale des affaires sociales (IGAS) diligentée pour comprendre les mécanismes qui ont conduit à ce décès, bien que n'ayant révélé aucune défaillance de la part du CPP qui avait évalué le protocole, a préconisé néanmoins que soient «mieux garanties l'indépendance et la qualité des travaux des CPP ${ }^{13} »$; la ministre des Affaires sociales et de la Santé a alors décidé d'instaurer ce tirage au sort.

Pour que la délibération du CPP soit valable, un quorum de sept membres doit être atteint, avec la présence requise par la loi d'au moins un méthodologiste et un représentant des patients. Le CPP s'assure tout d'abord de la recevabilité administrative du dossier soumis par le promoteur de l'étude et confirme ou non la catégorie dans laquelle il est présenté. Puis la justification et la méthodologie de l'étude sont revues, ainsi que tous les documents à destination des volontaires qui auront accepté d'y participer, notamment la note d'information et le formulaire de consentement que doivent signer les participants à l'étude. Depuis la mise en place des différents textes réglementaires assurant la protection des personnes au regard du traitement des données personnelles, le CPP doit s'assurer que les participants d'une étude restent maîtres du traitement des données les concernant et doit prévenir toute atteinte à leur vie privée. Dans le cas où l'examen d'une étude requiert une expertise absente d'un CPP, celle-ci peut être recherchée à l'extérieur du CPP.

\footnotetext{
7 Loi n 2004-806 du 9 août 2004 (J.0. du 11 août 2004) relative à la politique de santé publique. $81^{\text {er }}$ collège : personnes ayant une qualification et une expérience approfondie en matière de recherche biomédicale (médecins, notamment pédiatres, psychiatres) et personnes qualifiées en biostatistique ou épidémiologie; médecins généralistes; pharmaciens hospitaliers; infirmier $(e) s .2^{e}$ collège : personnes qualifiées en matière d'éthique; psychologues; travailleurs sociaux; personnes qualifiées en matière juridique ; représentants d'associations agréées de malades et d'usagers du système de santé.

9 www.cncpp.fr

10 https://www.lemonde.fr/sante/article/2016/10/11/essai-clinique-de-rennes-un-drame-en-cinqquestions_5011900_1651302.html

11 Décret 2016-1537 du 16 novembre 2016, JORF n 0267 du 17 novembre 2016, texte $n^{\circ} 27$.

12 Commission hébergée par la direction générale de la Santé.

13 https://www.igas.gouv.fr/spip.php?article531
}

Les CPP peuvent alors faire appel à des experts extérieurs, s'ils pensent que cela est nécessaire pour l'évaluation d'une recherche. Ces experts doivent rédiger un rapport dont il est tenu compte lors de l'analyse du dossier en séance.

\section{Les Comités de protection des personnes (CPP) dans l'espace européen}

Une grande disparité s'observe dans l'organisation des comités d'éthique de la recherche (CER) des pays de I'Union européenne ${ }^{14}$. Certains pays, tels que les Pays-Bas, ont adopté un système d'accréditation qui a entraîné une diminution importante du nombre de C\&R à travers le pays (d'environ 50 , ils sont passés à 24 ). La Belgique héberge quelques 215 CER, mais 22 fonctionnent comme nos CPP, alors que le Grand-Duché du Luxembourg ne s'est doté que d'un CहR qui exerce une fonction d'évaluation éthique des projets de recherche mais sert aussi de lieu de réflexion, comme le fait en France le Comité consultatif national d'éthique pour les sciences de la vie et de la santé $\left(\mathrm{CCN}^{15}\right)$. Quel que soit le modèle d'évaluation des essais cliniques en termes de protection des personnes, l'ensemble des CER de l'Union européenne doit appliquer la réglementation européenne, qui a récemment évolué fortement. En effet, pour éviter les trop grandes disparités entre les États membres de I'Union, les différentes directives, dont la transposition dans les cadres réglementaires nationaux peut varier d'un pays à l'autre, ont été remplacées par des règlements dont l'application obligatoire par les États membres ne nécessite pas de transposition. Ainsi, les directives sur les essais cliniques, sur les dispositifs médicaux et sur la protection des données personnelles (dont les données de santé) sont désormais remplacées par des règlements ${ }^{16}$. Des procédures coordonnées pour donner l'autorisation des essais cliniques ou des investigations cliniques des nouveaux dispositifs médicaux seront prochainement mises en place et déployées dans les 27 États membres. L'enjeu est de permettre à l'Union européenne de rester présente dans le marché très lucratif de la recherche biomédicale, en résistant à la concurrence des pays à coûts plus bas

\footnotetext{
14 Plus d'informations sur http://www.eurecnet.org/information/index.html

15 www.ccne-ethique.fr

16 - Règlement (UE) 2017/745 du Parlement et du Conseil du 5 avril 2017 relatif aux dispositifs médicaux, JOUE du 5 mai 2017, L117, pages 1-175. https://eur-lex. europa.eu/legal-content/FR/TXT/?uri=celex\%3A32017R0745

- Règlement (UE) 536/2014 du Parlement et du Conseil du 16 avril 2014 relatif aux essais cliniques des médicaments à usage humain, J0Uદ du 27 mai 2014, L158, page 1-67. https://eur-lex.europa.eu/legal-content/FR/TXT/PDF/?uri=CELEX:3201 4R0536\&from $=G A$

- Règlement (UE) 2016/679 du Parlement et du Conseil du 27 avril 2016 relatif à la protection des personnes physiques à l'égard du traitement des données à caractère personnel et à la libre circulation de ces données, JOUE du 4 mai 2016, L 119, p. 1-88 L. https://eur-lex.europa.eu/legal-content/FR/TXT/?uri=CELEX\%3A32016R0679
} 
comme l'Inde ou le Brésil, ou à celle de pays ayant des délais d'enregistrement plus rapides comme les États-Unis ou le Royaume-Uni.

\section{Le CPP, le maillon dérangeant ?}

Les dossiers de recherche clinique doivent être évalués selon un calendrier défini par la loi. Les CPP ont ainsi 45 jours pour rendre leur avis, si le dossier ne suscite pas de questions. Cependant, très fréquemment, des échanges avec les promoteurs sont nécessaires pour clarifier des points jugés incomplets, voire pour modifier certaines parties du dossier. Assez régulièrement, les promoteurs, notamment ceux appartenant à l'industrie pharmaceutique, représentés par leurs syndicats interprofessionnels ${ }^{17}$, prétendent que ces délais sont la cause de la perte d'attractivité de la France pour la recherche clinique ${ }^{18,19}$.

De même, certains parlementaires pointent l'absence de moyens (en personnels administratifs, en formations des membres, etc.) donnés aux CPP, qui serait une des causes du décrochage de la France dans le classement des pays attractifs en matière de recherche clinique ${ }^{20}$. Néanmoins, la Conférence nationale des CPP (CNCPP) a récemment montré que si l'organisation actuelle des CPP pouvait en effet être à l'origine de certains dépassements des délais requis, le temps de réponse des promoteurs aux questions posées après l'analyse de leurs dossiers contribuait également significativement à ces dépassements ${ }^{21}$. Après la délibération en séance, si cela s'avère nécessaire, le CPP envoie ses questions et demandes de modifications au promoteur de la recherche. Règlementairement, le chronomètre s'arrête alors, jusqu'au jour de la réception de ses réponses par le CPP. Le temps mis à répondre peut être très long, atteignant parfois plusieurs mois. La plupart des publications relatives aux délais d'émission de l'avis des CPP évoquent des délais globaux, qui comptabilisent donc dans le temps de travail des CPP, cette période de réponse des promoteurs, et ainsi, leur attribuent un retard qui ne leur est donc pas imputable.

Durant la récente pandémie de Covid-19, de nouvelles tensions sont apparues entre les CPP et leur tutelle administrative, la Direction générale de la santé. En effet, face à l'urgence sanitaire, notamment celle rencontrée lors des premières semaines de la pandémie, les CPP ont dû faire face à une multitude d'études cliniques à évaluer de manière accélérée, en quelques jours, au lieu des 45 ou 60 jours habituels en cas de questions soulevées par un CPP. Dans le souci constant de protection des participants à ces études, les CPP ont émis des avis parfois très réservés sur un certain nombre d'essais, souvent élaborés trop vite pour faire face à une situation sanitaire catastrophique. Le taux d'avis défavorables a atteint les $12 \%$ alors qu'il est généralement autour de $8 \%$. Les CPP se

17 Le LEEM pour les industries pharmaceutiques, le SNITEM pour les industries du dispositif médical ou I'AFCRO pour les prestataires de services en recherche clinique.

18 www.leem.org/sites/default/files/2018-12/Complet2018_Attractivité-France-Recherche-Clinique.pdf 19 «L'activité de recherche industrielle, qui constitue le reflet le plus direct de l'attractivité de la France, reste en difficulté. Les nombreuses modifications règlementaires intervenues en 2018, avec leurs corollaires de délais de mise en place, ont probablement eu un impact négatif sur le lancement de nouvelles études, en particulier dans le cas des études interventionnelles qui sont soumises à l'avis des Comités de prévention des personnes (CPP) ». https://www.afcros.com/groupes-de-travail/attractivite/

20 https://www.assemblee-nationale.fr/dyn/15/rapports/cion-soc/l15b0908_rapport-fond\#

21 Rencontre 2020 phases précoces en cancérologie, 26 novembre 2020, https://virtualevent.olimpe.com/

22 Webinaire CNCP, lundi 16 octobre 2020, site de la Conférence Nationale des CPP, https://cncpp.fr/ sont vus alors exposés à de vives critiques pour avoir simplement appliqué les termes de la Déclaration d'Helsinki, texte de référence internationale en matière d'éthique de la recherche biomédicale $e^{23}$, et pour avoir respecté les principes affirmés dans la Convention d'Oviedo ${ }^{24}$, ratifiée par la France en 2012, qui imposent qu' « une recherche médicale impliquant des êtres humains ne peut être conduite que si l'importance de l'objectif dépasse les risques et inconvénients pour les personnes impliquées ».

\section{Conclusion}

En conclusion, si une certaine lassitude gagne parfois les membres des Comités de protection des personnes (CPP) qui acceptent parfois difficilement la remise en cause de leur travail bénévole par ces fréquentes critiques les rendant responsables de la perte d'attractivité de la recherche clinique française, soulignons que tous poursuivent cette activité, car ils ont conscience des rôles éminemment social et éthique qu'ils jouent, en contribuant à la protection des personnes qui participent aux recherches biomédicales.

Les CPP sont le maillon essentiel de la confiance qui doit régner entre les participants à ces recherches et leurs opérateurs. Cette confiance, écornée pendant la période de crise sanitaire que nous vivons, est capitale pour assurer le développement de la recherche clinique en France. L'arrivée espérée de nouveaux experts, qui doivent contacter les Agences régionales de santé pour participer à la vie des Comité de protection des personnes, permettra au système français d'encadrement de la recherche clinique de continuer à offrir cette très grande protection à ses participants que de nombreux pays nous envient. $\diamond$

The Committees for the Protection of Persons:

The ethical link in the supervision of French clinical research

\section{LIENS D’INTÉRÊT}

Les auteurs déclarent n'avoir aucun lien d'intérêt concernant les données publiées dans cet article.

\section{TIRÉS À PART}

F. Hirsch

\footnotetext{
23 « Une recherche médicale impliquant des êtres humains ne peut être conduite que si l'importance de l'objectif dépasse les risques et inconvénients pour les personnes impliquées » : https://www.wma.net/fr/policies-post/declaration-dhelsinki-de-lamm-principes-ethiques-applicables-a-la-recherche-medicale-impliquant-des-etres-humains/.

24 « Aucune recherche ne peut être entreprise sur une personne à moins que les conditions suivantes ne soient réunies... (2) les risques qui peuvent être encourus par la personne ne sont pas disproportionnés par rapport aux bénéfices potentiels de la recherche ». Conseil de l'Europe, Convention pour la protection des Droits de l'Homme et de la dignité de l'être humain à l'égard des applications de la biologie et de la médecine, STE $n^{\circ} 164$, Oviedo, 04/04/1997, article 16, ratifiée par la France le $1^{\text {er }}$ avril 2012. https://rm.coe.int/090000168007 cf99.
} 